\title{
Expression of the mitotic-arrest deficiency 2 is associated with chemotherapy resistance in ovarian serous adenocarcinoma
}

\author{
YUSUKE NAKANO, TOSHIYUKI SUMI, MASATOMO TERAMAE, MASANARI MORISHITA, \\ TAKESHI FUKUDA, HIROYUKI TERADA, HIROYUKI YOSHIDA, \\ YOSHINARI MATSUMOTO, TOMOYO YASUI and OSAMU ISHIKO \\ Department of Obstetrics and Gynecology, Osaka City University Graduate \\ School of Medicine, Osaka 545-8585, Japan
}

Received March 15, 2012; Accepted June 8, 2012

DOI: $10.3892 /$ or.2012.1907

\begin{abstract}
Mitotic-arrest deficiency 2 (MAD2) is a key component of spindle assembly checkpoint (SAC) function; SAC mediates spindle microtubule attachment to kinetochores on chromosomes and chromosomal segregation during mitosis. To determine whether MAD2 expression is associated with chemotherapy resistance in ovarian serous adenocarcinoma, we reviewed tumor samples from 41 cases of ovarian serous adenocarcinoma at Osaka City University Medical School Hospital (Osaka, Japan), 2000-2007. Of the 41 cases, 24 were recurrent and 17 were not recurrent. Expression of MAD2 was investigated in paraffin-embedded sections using a MAD2 antibody. Quantitative analysis of MAD2 expression gave mean weighted scores of 4.3 for the relapsed group and 7.2 for the relapse-free group; the expression was, thus, significantly greater in the relapse-free group compared to the relapsed group $(\mathrm{P}=0.023)$. When the 41 cases were classified into lowand high-expression, these classifications showed no significant difference in terms of progression-free survival $(\mathrm{P}=0.0685)$, however, overall survival for the low-expression group was significantly shorter than that of the high-expression group $(\mathrm{P}=0.0188)$. The present study implies that MAD2 expression levels can indicate sensitivity to anticancer agents, and risk for recurrence.
\end{abstract}

\section{Introduction}

Ovarian epithelial carcinoma is classified into four morphologically distinct categories: serous, mucinous, endometrioid and clear-cell. Serous adenocarcinoma accounts for $\sim 50 \%$ of all ovarian carcinoma (1). Ovarian serous adenocarcinoma cases $(\sim 70 \%)$ have progressed into the abdominal cavity at the

Correspondence to: Dr Toshiyuki Sumi, Department of Obstetrics and Gynecology, Osaka City University Graduate School of Medicine, 1-4-3 Asahimachi, Abeno-ku, Osaka 545-8585, Japan E-mail: sumi-toshi@med.osaka-cu.ac.jp

Key words: mitotic-arrest deficiency 2, chemotherapy resistance, chromosomal instability, serous adenocarcinoma, ovary time of diagnosis, giving them a stage III classification, based on the International Federation of Gynecology and Obstetrics (FIGO) criteria. As it is difficult to completely remove most such tumors by surgery, response to chemotherapy determines their prognosis. Although ovarian serous adenocarcinoma is highly sensitive to chemotherapy, many cases relapse. Corrected actuarial survival rates are $41.0 \%$ at 5 years for all stages of ovarian serous adenocarcinoma, 35.1\% for stage III, and $19.2 \%$ for stage IV (1). Reduction of recurrence for patients with partial or complete response to chemotherapy would greatly improve their prognoses.

Mitotic-arrest deficiency 2 (MAD2) was the first gene of the mammalian mitotic spindle checkpoint pathway to be characterized (2). MAD2 localizes at kinetochores after chromosome condensation and before anaphase (3); it significantly affects the transition from metaphase to anaphase by inhibiting the anaphase-promoting complex/cyclosome (APC/C). This process ensures that the chromosomes are correctly aligned at the metaphase plate prior to daughter-cell segregation (4,5). Therefore, MAD2 is a key component of the mitotic spindle checkpoint pathway, which plays a crucial role in preventing loss or gain of chromosomes within cells (6). A compromised mitotic spindle checkpoint results in an abnormal number of chromosomes, known as chromosomal instability (CIN) (7). CIN is characterized by alterations in chromosome number and is commonly detected as aneuploidy $(8,9)$; it has been reported in most types of human cancer. Although the underlying molecular mechanisms are unclear, MAD2 overexpression in transgenic mice notably results in CIN and initiates carcinogenesis in a wide variety of tumors (10). Some studies suggest that a compromised mitotic spindle checkpoint, through MAD2 overexpression, is a significant step in malignancy progression; in fact, MAD2 overexpression is observed in several cancers (11-15).

We have previously reported that MAD2 overexpression occurs in most cases of mucinous ovarian carcinomas and that it is potentally correlated to mucinous ovarian carcinogenesis (16). However, MAD2 overexpression alone may not be sufficient for mucinous ovarian carcinogenesis. Some studies suggest a correlation between MAD2 overexpression and various clinicopathological characteristics, such as histological grade (differentiation), metastasis and prognosis 
$(11,12,14,15)$. However, reduced MAD2 expression occurs in some human cancers $(4,17)$ and is associated with in vitro resistance to chemotherapies that use microtubule-targeting agents or DNA-damaging agents $(18,19)$. In this study, we examined the relationship between MAD2 expression and chemotherapy resistance in ovarian serous adenocarcinoma.

\section{Materials and methods}

Patients and samples. We initially treated 63 cases of ovarian serous adenocarcinoma at Osaka City University Medical School Hospital (Osaka, Japan), between 2000 and 2007. In this study, we reviewed 41 cases in which maximum debulking surgery and platinum-based anticancer drugs as first-line chemotherapy were used, and from patients who had partial or complete responses from this chemotherapy. Tumor samples were obtained following primary surgery. Written informed consent was obtained from all patients prior to immunohistochemical examination. This study was approved by the Ethics Committee of Osaka City University (IRB no. 2201).

Of the 41 cases, 24 were recurrent (relapsed group), and 17 were disease-free (relapse-free group). Based on the FIGO criteria, 1 case in the relapsed group was classified as stage I, 1 as stage II, 19 as stage III and 3 as stage IV. In the relapse-free group, 3 cases were classified as stage I, 0 as stage II, 14 as stage III and 0 as stage IV (Table I).

Immunohistochemical analysis. The expression of MAD2 was investigated in paraffin-embedded sections, using a MAD2 antibody and the avidin-biotin peroxidase complex method. Paraffin sections $(4-\mu \mathrm{m})$ were de-paraffinized and immersed in $3 \%$ hydrogen peroxidase in methanol to block endogenous peroxidase activity. Next, an antigen retrieval procedure was performed by immersing the slides in $10 \mathrm{mM}$ citrate buffer (pH 6.0) and heating in an autoclave at $110^{\circ} \mathrm{C}$ for $20 \mathrm{~min}$. The sections were then washed in phosphate-buffered saline (PBS). The protocol for the Dako LSAB 2 peroxidase kit (Dako, Kyoto, Japan) was followed. The sections were incubated with the primary antibodies for $2 \mathrm{~h}$ at room temperature. The primary antibody used for this study was monoclonal rabbit anti-human MAD2 (1:200; Proteintech Group, Inc., Chicago, IL, USA). Sections were rinsed with PBS for $15 \mathrm{~min}$ and incubated for $10 \mathrm{~min}$ with secondary antibody (biotinylated goat anti-mouse and rabbit immunoglobulin G; Dako). Sections were then incubated with the streptavidin-peroxidase complex; 3,3'-diaminobenzidine was used as chromogen. Sections were then counterstained with Mayer's hematoxylin. Specificity of immunohistochemical reactions was checked by omitting the primary antibody. Quantitative analysis of MAD2 expression was based on the scoring method of Sinicrope et al (20). Briefly, mean percentages of positive tumor cells were determined in five separate areas (magnification, $\mathrm{x} 400$ ) and assigned to the following values: $0,<5 \% ; 1,5-25 \% ; 2,25-50 \%$; $3,50-75 \%$ or $4,>75 \%$. Immunostaining intensity was scored as: $1+$, weak; $2+$, moderate or $3+$, intense. For each specimen, percentage of positive tumor cells was multiplied by staining intensity to produce a weighted score.

Statistical analysis. Kaplan-Meier and log-rank tests were used for the prognostic analyses. StatView 5.0 (Abacus Concepts,
Table I. Patient characteristics.

\begin{tabular}{lccc}
\hline & Relapsed & Relapse-free & P-value \\
\hline No. of cases & 24 & 17 & \\
Age & & & NS \\
Mean & 56.0 & 53.2 & \\
Range & $36-82$ & $26-73$ & \\
FIGO stage & & & NS \\
I & 1 & 3 & \\
II & 1 & 0 & \\
III & 19 & 14 & \\
IV & 3 & 0 & NS \\
Chemotherapy & & & \\
Taxane + carboplatin & 20 & 15 & \\
Platinum only & 4 & 2 & \\
\hline
\end{tabular}

FIGO, International Federation of Gynecology and Obstetrics; taxane, paclitaxel or docetaxel; platinum only, cisplatin or carboplatin only; NS, none significance.

Berkeley, CA, USA) was used for data analysis and $\mathrm{P}<0.05$ was considered significant. Continuous variables were expressed as mean \pm standard deviation $(\mathrm{SD})$ or mean \pm standard error (SE), as shown in the figures. Weighted scores were compared using the Mann-Whitney U test.

\section{Results}

Patient characteristics. The mean age was 56.0 years (range, 36-82) in the relapsed group and 53.2 years (range: 26-73) in the relapse-free group. No significant difference was observed among the two groups (Table I). All patients recieved platinumbased chemotherapy, which was mostly taxane (paclitaxel or docetaxel)/carboplatin.

MAD2 expression. MAD2 was expressed in tumor cell nuclei (Fig. 1). Mean weighted scores were 4.3 in the relapsed and 7.2 in the relapse-free group (Table II), and were thus significantly greater in the relapse-free than in the relapsed group (Fig. 2).

Survival. In the relapsed group, mean progression-free survival was 18.8 months (range, 3-49). Mean overall survival was 36.7 months (range, 8-66) for the relapsed group and 73.9 months (range, 46-119) for the relapse-free group.

Grade of MAD2 expression. The 41 cases were re-classified as showing low or high MAD2 expression. The low expression group contained 24 cases with weighted scores from 0-6 and the high expression group contained 17 cases with weighted scores from 8-12. The mean age of the low expression group was 57.1 years (range, 26-82) and that of high expression group was 50.4 years (range, 34-73). Based on the FIGO criteria, 3 low-expression cases were classified as stage I, 1 as stage II, 20 as stage III and 3 as stage IV; among high-expression cases, 1 was classified as stage I, 0 as stage II, 13 as stage III and 0 as stage IV. In the low-expression group, 19 patients relapsed and 

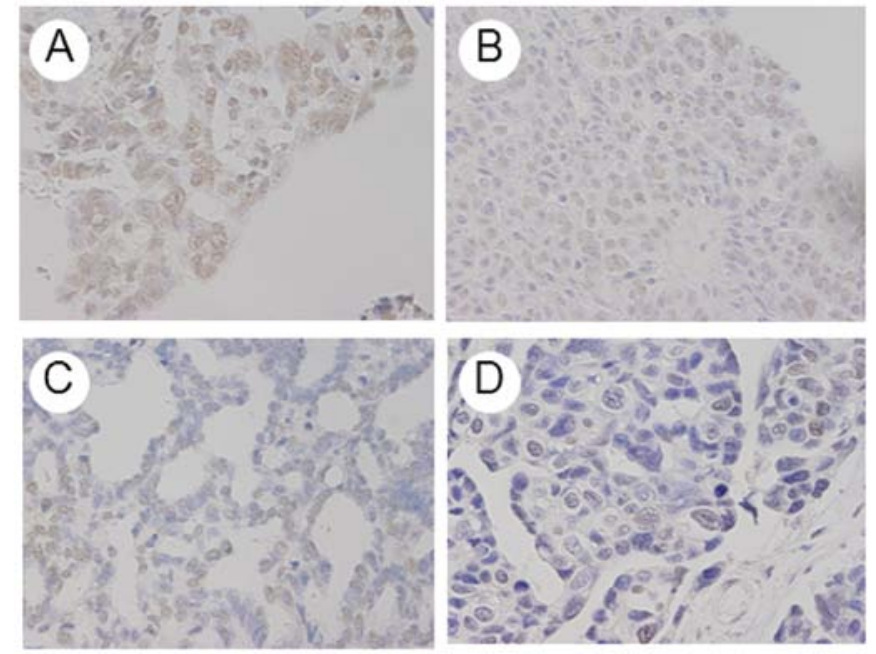

Figure 1. Immunohistochemical staining of MAD2 in ovarian serous adenocarcinoma. (A) Relapse-free group, score 12; (B) relapsed group, score 8; (C) relapsed group, score 4; (D) relapsed group score 0 (A-D, magnification, $\mathrm{x} 400$ ). MAD2 was expressed in nuclei of tumor cells. Hematoxylin and eosin stain.

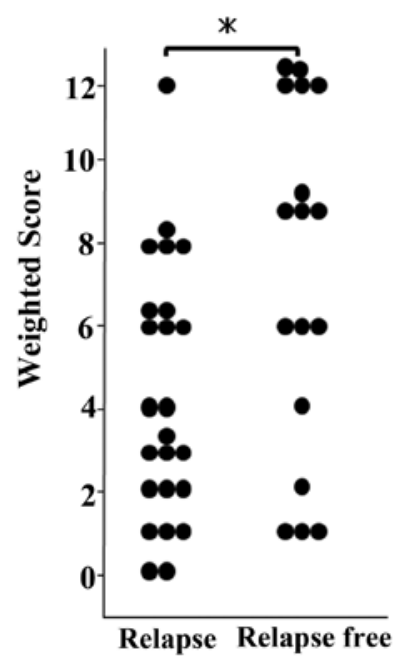

Figure 2. Weighted score for MAD2 in ovarian serous adenocarcinoma MAD2 expression was significantly greater in the relapse-free group than in the relapsed group. ${ }^{*} \mathrm{P}=0.023$ (Mann-Whitney $\mathrm{U}$ test).

17 died; in the high-expression group, 5 patients relapsed and 3 died (Table III). There was no significant difference in the estimated mean progression-free survival between the lowexpression group (47.6 months) and the high-expression group (67.1 months) $(\mathrm{P}=0.0685$; Fig. 3). However, overall estimated mean survival was significantly shorter for the low-expression (61.7 months) than for the high-expression group ( 82.0 months) ( $\mathrm{P}=0.0188$; Fig. 4).

\section{Discussion}

MAD2 is a key component of the mitotic spindle checkpoint pathway, which if compromised, can result in CIN and tumorigenesis. MAD2 overexpression has been shown to promote aneuploidy, tumorigenesis and tumor progression (21). Reportedly, MAD2 overexpression is a critical mediator
Table II. Weighted scores in relapsed and relapse-free groups.

\begin{tabular}{lccc}
\hline Weighted-score & Relapsed (n) & Relapse-free (n) & Total (n) \\
\hline 0 & 2 & 0 & 2 \\
1 & 3 & 3 & 6 \\
2 & 3 & 1 & 4 \\
Total, n (\%) & $8(33.3)$ & $4(23.5)$ & $12(29.3)$ \\
3 & 4 & 0 & 4 \\
4 & 2 & 1 & 3 \\
6 & 5 & 3 & 8 \\
Total, n (\%) & $11(45.8)$ & $4(23.5)$ & $15(36.6)$ \\
8 & 4 & 0 & 4 \\
9 & 0 & 4 & 4 \\
12 & 1 & 5 & 6 \\
Total, n (\%) & $5(20.8)$ & $9(52.9)$ & $14(34.1)$ \\
Weighted score, & & & \\
mean & 4.3 & 7.2 & 5.5 \\
\hline
\end{tabular}

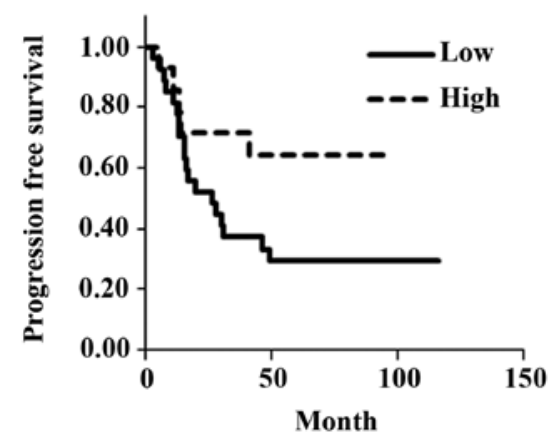

Figure 3. Progression-free survival according to level of MAD2 expression. The low expression group (weighted score, 0-6) contained 24 cases and the high expression group (weighted score, 8-12) contained 17 cases. Relapse rates were significantly different between the low expression group, with 19 cases $(70.3 \%)$ relapsed and the high expression group with 5 cases $(35.7 \%)$, but these groups showed no significant difference in progression-free survival ( $\mathrm{P}=0.0685$; Kaplan-Meier and log-rank tests).

of CIN, as seen in inactivation of p53 and Rb pathways (22). MAD2 overexpression has also been observed in some human cancers (11-15). A correlation has been implied between MAD2 overexpression and various clinicopathological characteristics, such as histological grade (differentiation), metastasis and prognosis $(11,12,14,15)$. In a study that assessed incidence of metastasis and survival time in 48 cases of human osteosarcoma, MAD2 overexpression was associated with early metastasis and poor prognosis (14). Another study showed MAD2 overexpression to be associated with lung cancer recurrence in mice (23).

In the present study, 39 of 41 samples $(95.2 \%)$ were stained positively for MAD2. Of the 41 cases, 24 were recurrent and 17 disease-free (Table I). Mean weighted scores for MAD2 expression were significantly less for the relapsed (4.3) than for the relapse-free group (7.2) (Fig 2; Table II). When the 41 cases were classified into 24 low-expression (weighted score, 0-6) and 17 high-expression (weighted score, 8 -12) cases (Table III), they 
Table III. Clinical background of cases with low and high expression of MAD2.

\begin{tabular}{|c|c|c|c|}
\hline & $\begin{array}{c}\text { Low expression } \\
(\text { score: } 0,1,2,3,4,6)\end{array}$ & $\begin{array}{l}\text { High expression } \\
\text { (score: } 8,9,12 \text { ) }\end{array}$ & P-value \\
\hline No. of cases & 27 & 14 & \\
\hline Age & & & NS \\
\hline Mean & 57.1 & 50.4 & \\
\hline Range & $26-82$ & $34-73$ & \\
\hline FIGO stage, $\mathrm{n}$ & & & NS \\
\hline $\mathrm{I}$ & 3 & 1 & \\
\hline II & 1 & 0 & \\
\hline III & 20 & 13 & \\
\hline IV & 3 & 0 & \\
\hline Relapses, n(\%) & $19(70.3)$ & $5(35.7)$ & $<0.05$ \\
\hline Deaths, $\mathrm{n}(\%)$ & $17(63.0)$ & $3(21.4)$ & $<0.05$ \\
\hline
\end{tabular}

FIGO, International Federation of Gynecology and Obstetrics; NS, not significant.

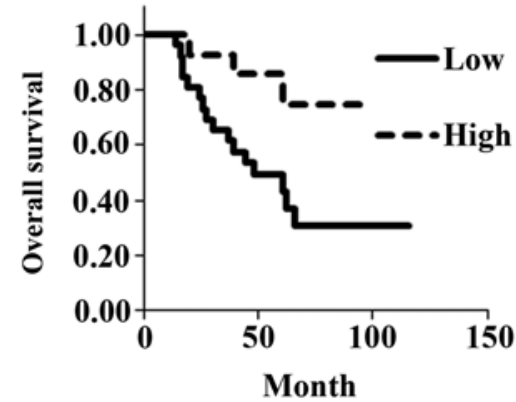

Figure 4. Overall survival according to MAD2 expression levels. The low expression group (weighted score, 0-6) contained 24 cases and the high expression group (weighted score, 8-12) contained 17 cases. In the lowexpression group, 17 cases were dead of disease $(63.0 \%)$ and 3 cases with the high-expression group were dead of disease (21.4\%). Death rates differed significantly between the low- and high-expression groups. In addition, there was a significant difference between the low- and high-expression groups in terms of overall survival ( $\mathrm{P}=0.0188$; Kaplan-Meier and log-rank tests).

showed no significant difference in progression-free survival, but overall survival for the low-expression group was significantly shorter for the high-expression group (Figs. 3 and 4). In this study, $87.8 \%(36 / 41)$ of cases were in advanced stages. However, the above-mentioned study (14) indicated significant association between MAD2 expression and clinical staging, which implies that poor prognoses reflect clinical staging rather than MAD2 expression. Adjuvant chemotherapy in our study could have affected prognoses, implying a relationship between MAD2 expression and chemotherapy resistance.

MAD2 is a key component of spindle assembly checkpoint (SAC) function which meditates attachment of spindle microtubules to kinetochores and accurate chromosomal segregation during mitosis $(24,25)$. One review focused on chemoresistance to taxane treatment in breast cancer, particularly in relation to the SAC and dysfunctional regulation of apoptotic signaling (26). Reportedly, cellular senescence induced by the low MAD2 expression affects paclitaxel sensitivity (27). Low MAD2 expression is also reportedly associated with resistance to paclitaxel in ovarian cancer cells (28) and gastric cancer cells (29), and Bcl-2 may be involved in this process. While failure of SAC function does not fully explain chemotherapy-resistant acquisition, it appears to take an important role. By extension, MAD2 expression level could be a predictor of chemoresistance. Our previous study suggested MAD2 expression to predict efficacy of neoadjuvant chemotherapy for locally advanced uterine cervical cancer (30). In this study, while progression-free survival between groups expressing low and high levels of MAD2 did not significantly differ ( $\mathrm{P}=0.0685$; Fig. 3), those expressing lower MAD2 levels had significantly shorter overall survival ( $\mathrm{P}=0.0188$; Fig. 4). Therefore, MAD2 expression may be an important predictor of prognosis for ovarian serous adenocarcinoma.

In conclusion, the results of the present study suggest that levels of MAD2 expression can help predict sensitivity to anticancer agents and risk of recurrence for these patients.

\section{Acknowledgements}

We thank the gynecologists at Osaka City University Medical School Hospital for their support (Osaka, Japan). This study was supported by the Osaka Medical Research Foundation for Incurable Diseases.

\section{References}

1. Heintz AP, Odicino F, Maisonneuve P, et al: Carcinoma of the ovary. FIGO 26th annual report on the results of treatment in gynecological cancer. Int J Gynecol Obstet 95: S161-S192, 2006.

2. Hardwick KG: Checkpoint signaling: Mad2 conformers and signal propagation. Curr Biol 15: R122-R124, 2005.

3. Lopez-Girona A, Furnari B, Mondesert O and Russell P: Nuclear localization of Cdc25 is regulated by DNA damage and a 14-3-3 protein. Nature 397: 172-175, 1999.

4. Wang X, Jin DY, Ng RW, Feng H, Wong YC, Cheung AL and Tsao SW: Significance of MAD2 expression to mitotic checkpoint control in ovarian cancer cells. Cancer Res 62: 1662-1668, 2002.

5. Li R and Murray A: Feedback control of mitosis in budding yeast. Cell 66: 519-531, 1991.

6. Orr-Weaver TL and Weinberg RA: A checkpoint on the road to cancer. Nature 392: 223-224, 1998. 
7. Yoon DS, Wersto RP, Zhou W, Chrest FJ, Garret ES, Kwon TK and Gabrielson E: Variable levels of chromosomal instability and mitotic spindle checkpoint defects in breast cancer. Am J Pathol 161: 391-397, 2002.

8. Lengauer C, Kinzler KW and Vogelstein B: Genetic instability in colorectal cancer. Nature 386: 623-627, 1997.

9. Lengauer C, Kinzler KW and Vogelstein B: Genetic instability in human cancer. Nature 396: 643-649, 1998

10. Sotillo R, Hernando E, Diaz-Rodriguez E, Teruya-Feldstein J, Cordon-Cardo C, Lowe SW and Benezra R: Mad2 overexpression promotes aneuploidy and tumorigenesis in mice. Cancer Cell 11: 9-23, 2007.

11. Li GQ, Li H and Zhang HF: Mad2 and p53 expression profiles in colorectal cancer and its clinical significance. World J Gastroenterol 9: 1972-1975, 2003.

12. Wang L, Yin F, Du Y, Du W, Chen B, Zhang Y, Wu K, Ding J, Liu J and Fan D: MAD2 as a key component of mitotic checkpoint: A probable prognostic factor for gastric cancer. Am J Clin Patho 131: 793-801, 2009.

13. Chen X, Cheung ST, So S, et al: Gene expression patterns in human liver cancers. Mol Biol Cell 13: 1929-1939, 2002.

14. Yu L, Guo WC, Zhao SH, Tang J and Chen JL: Mitotic arrest defective protein 2 expression abnormality and its clinicopathologic significance in human osteosarcoma. APMIS 118: 222-229, 2010

15. Zhang SH, Xu AM, Chen XF, Li DH, Sun MP and Wang YJ: Clinicopathologic significance of mitotic arrest defective protein 2 overexpression in hepatocellular carcinoma. Hum Pathol 39: 1827-1834, 2008.

16. Nakano Y, Sumi T, Morishita M, Fukuda T, Nobeyama H, Yoshida H, Matsumoto Y, Yasui T and Ishiko O: Mitotic arrest deficiency 2 induces carcinogenesis in mucinous ovarian tumors. Oncol Lett 3: 281-286, 2012.

17. Wang X, Jin DY, Wong YC, Cheung AL, Chun AC, Lo AK, Liu Y and Tsao SW: Correlation of defective mitotic checkpoint with aberrantly reduced expression of MAD2 protein in nasopharyngeal carcinoma cells. Carcinogenesis 21: 2293-2297, 2000.

18. Fung MK, Cheung HW, Ling MT, Cheung AL, Wong YC and Wang X: Role of MEK/ERK pathway in the MAD2-mediated cisplatin sensitivity in testicular germ cell tumor cells. Br J Cancer 95: 475-484, 2006.

19. Cheung HW, Chun AC, Wang Q, Deng W, Hu L, Guan XY, Nicholls JM, Ling MT, Chuan Wong Y, Tsao SW, et al: Inactivation of human MAD2B in nasopharyngeal carcinoma cells leads to chemosensitization to DNA-damaging agents. Cancer Res 66: 4357-4367, 2006.
20. Sinicrope FA, Ruan SB, Cleary KR, Stephens LC, Lee JJ and Levin B: Bcl-2 and p53 oncoprotein expression during colorectal tumorigenesis. Cancer Res 55: 237-241, 1995.

21. Wu CW, Chi CW and Huang TS: Elevated level of spindle checkpoint MAD2 correlates with cellular mitotic arrest, but not with aneuploidy and clinicopathological characteristics in gastric cancer. World J Gastroenterol 10: 3240-3244, 2004.

22. Schvartzman JM, Duijf PH, Sotillo R, Coker C and Benezra R: Mad2 is a critical mediator of the chromosome instability observed upon $\mathrm{Rb}$ and $\mathrm{p} 53$ pathway inhibition. Cancer Cell 19: 701-714, 2011.

23. Sotillo R, Schvartzman JM, Socci ND and Beneztra R: Mad2induced chromosome instability leads to lung tumor relapse after oncogene withdrawal. Nature 464: 436-440, 2010.

24. Zhou J, Yao J and Joshi HC: Attachment and tension in the spindle assembly checkpoint. J Cell Sci 115: 3547-3555, 2002.

25. Buke DJ and Stukenberg PT: Linking kinetochoremicrotubule binding to the spindle checkpoint. Dev Cell 14: 474-479, 2008.

26. McGrogan BT, Gilmartin B, Carney DN and McCann A: Taxanes, microtubules and chemoresistant breast cancer. Biochim Biophys Acta 1785: 96-132, 2008.

27. Prencipe M, Fitzpatrick P, Gorman S, Tosetto M, Klinger R, Furlong F, Harrison M, O'Connor D, Roninson IB, O'Sullivan J and McCann A: Cellular senescence induced by aberrant MAD2 levels impacts on paclitaxel responsiveness in vitro. Br J Cancer 101: 1900-1908, 2009.

28. Hao X, Zhou Z, Ye S, Zhou T, Lu Y, Ma D and Wang S: Effect of Mad2 on paclitaxel-induced cell death in ovarian cancer cells. J Huazhong Univ Sci Technolog Med Sci 30: 620-625, 2010.

29. Du Y, Yin F, Liu C, Hu S, Qang J, Xie H, Hong L and Fan D: Depression of Mad2 inhibits apoptosis of gastric cancer cells by upregulating $\mathrm{Bcl}-2$ and interfering mitochondrion pathway. Biochem Biophys Res Commun 345: 1092-1098, 2006.

30. Morishita M, Sumi T, Nakano Y, Fukuda T, Nobeyama H, Yoshida H, Matsumoto Y, Yasui T and Ishiko O: Expression of mitotic-arrest deficiency 2 predicts the efficacy of neoadjuvant chemotherapy for locally advanced uterine cervical cancer. Exp Ther Med 3: 341-346, 2012. 